\title{
Network Dimensioning with Carrier Aggregation
}

\author{
Emir Kavurmacioglu \\ Division of Systems Engineering \\ Boston University \\ Boston, MA 02215 \\ emir@bu.edu
}

\author{
David Starobinski \\ Division of Systems Engineering \\ Boston University \\ Boston, MA 02215 \\ staro@bu.edu
}

\begin{abstract}
A recent policy ruling by the Federal Communications Commission (FCC) set aside a fixed amount of cleared spectrum for smaller network providers. Thanks to this ruling, smaller providers can improve their quality of service using carrier aggregation. In this paper, we determine the optimal (minimum) level of carrier aggregation that a smaller provider needs in order to bring its service in line with a larger provider in the same market. Toward this end, we provide an asymptotically exact formula for the loss (blocking) probability of flows under a quality-driven $(Q D)$ regime. Using this formula, we establish an efficient way of numerically calculating the optimal level of carrier aggregation and derive scaling laws. Specifically, we show that the optimal level of carrier aggregation scales sub-linearly with respect to the scaling factor, i.e., the ratio between the network capacities of the two providers, and decreases with the initial traffic load of the providers. We derive a closed-form linear upper bound on the optimal level of carrier aggregation and prove that it is the tightest possible. We provide numerical results, showing the accuracy of our methods and illustrating their use. We also discuss the extension of our results to delayrelated metrics as well as their application to profitable pricing in secondary spectrum markets.
\end{abstract}

\section{INTRODUCTION}

In recent years, the U.S. Federal Communications Commission (FCC) has made stringent efforts to clear spectrum bands and reallocate them for more efficient use. In particular, to preserve the competitive landscape of the wireless industry, the FCC has recently decided to set aside $30 \mathrm{MHz}$ of spectrum for service providers that hold less than a third of the spectrum in a specific market [1,2]. With the $600 \mathrm{MHz}$ spectrum auction on the horizon, this ruling is poised to have a significant impact on the industry [3]. The ruling has already caused some controversy in the market as it restricts the amount of spectrum larger providers have access to [4], though some public interest groups are asking for it to be increased to $40 \mathrm{MHz}$ [5].

The policy ruling is facilitated by a central feature of LTE-Advanced networks (as defined in 3GPP Release 10 and beyond) called carrier aggregation [6-9]. Car- rier aggregation allows service providers to aggregate contiguous or non-contiguous component carriers up to $100 \mathrm{MHz}$ total bandwidth. This significantly improves the performance of the network compared to LTE specifications defined in Release 8 [10], where the maximum supported bandwidth is $20 \mathrm{MHz}$.

A significant challenge associated with the ruling is to identify how much additional spectrum a smaller provider needs to improve its service to the level of a larger provider, which initially holds a competitive advantage in the market due to economies of scale. If this criterion is met, the spectrum reservation policy effectively fosters a competitive market. Otherwise, the policy inherently risks wasting highly valuable spectrum.

The main goal of this paper is to determine the optimal (minimum) level of carrier aggregation that a smaller provider needs to bring its quality of service in line with a larger provider operating in the same market. Furthermore, we aim to provide insight into the relationships between the optimal level of carrier aggregation and fundamental network parameters, such as the traffic load and capacity.

This paper makes several contributions. First, we propose an asymptotically exact approximation of the Erlang-B blocking formula under a quality-driven ( $Q D)$ regime that holds for large traffic and network capacities [11]. We refer to this approximation as the $Q D$ formula. As explained in the sequel, this formula is applicable to both voice and data traffic models. Since the Erlang-B formula does not easily yield itself to mathematical analysis, the QD formula is useful to provide more explicit insight into the impact of network parameters. All the subsequent results derived in the paper are based on the QD formula and several numerical examples are provided to demonstrate the accuracy of the results for typical network parameters.

Second, we identify the optimal carrier aggregation decision for the smaller provider through which the mar- 
ket outcome becomes favorable. We provide an efficient method for numerically calculating the optimal level of carrier aggregation.

Third, we derive scaling laws on optimal carrier aggregation with respect to the scaling factor, i.e. the ratio of the capacity of the larger provider to that of the smaller provider, and establish a sub-linear relationship. We also prove that while the level of carrier aggregation needed increases with the scaling factor, it decreases when the initial traffic load of the providers gets higher.

Fourth, we establish concavity properties and derive the tightest possible linear upper bound on optimal carrier aggregation as a simple and explicit function of the network parameters and the scaling factor. We further propose a numerical procedure to compute a piecewise linear bound. We provide numerical examples to illustrate applications of our results in cellular markets.

Finally, we extend our results to delay-related metrics (i.e., based on the Erlang-C formula) and discuss the application of our results to the profitable pricing of secondary users in a dynamic spectrum sharing scenario.

The rest of the paper is organized as follows. In Section II, we survey previous work. In Section III, we introduce our model. Next, in Section IV, we propose a many-server approximation of the Erlang-B formula under the QD regime. We then use this approximation in Section $\mathrm{V}$ to identify the impact of reserved spectrum through the analysis of optimal carrier aggregation, in which we provide numerical calculation methods, structural properties, and explicit general bounds. We also provide extension of our results to delay systems as well as pricing in secondary spectrum markets. We conclude the paper in Section VI.

\section{RELATED WORK}

In this section, we survey previous work on manyserver approximations of queuing systems, carrier aggregation, and spectrum markets. We highlight the differing contributions of our paper at the end.

The many-server approximation that forms the basis of the QD regime was first introduced in Iglehart's work [12]. The paper considers a setting where the arrival rate and the number of servers both become very large and the ratio of the arrival rate to the service rate (i.e., the traffic load) is a constant that is strictly smaller than one. Under proper statistical assumptions, the process describing the evolution of the queue occupancy converges to a Ornstein-Uhlenbeck diffusion process. Halfin and Whitt [13] provide another many-server approximation that characterizes queues in a quality-andefficiency driven (QED) regime, i.e., where the arrival rate and the number of servers both become very large and the traffic load approaches one. The work of Zeltyn and Mandelbaum [14] provides an overview of different types of many-server approximations and is useful as a general reference. In our work, we utilize the QD regime approximations that allow us to analyze the quality of service experienced by voice calls and data flows in cellular networks.

Carrier aggregation has been gaining significant attention since it has been introduced in 3GPP Release 10 on LTE-Advanced in 2011. Several papers in the literature explain practical considerations to achieve desired performance levels in networks, such as deployment options, implementation frameworks, and challenges in the physical layer [6-8]. The work by Shen et al. [15] provides an overview on all layers, while also underlining the interest of several major U.S. providers in the technology. Alotaibi and Sirbu provide a comprehensive cost benefit analysis of spectrum aggregation in [16]. A recent paper by Doyle et al. [9] introduces an interesting application of carrier aggregation. The authors consider the possible uses of carrier aggregation in a dynamic spectrum access, such as dynamically aggregating carriers to address coverage or congestion issues. They also propose a regulatory framework that supports this enhanced form of carrier aggregation.

Fungibility of the aggregated spectrum is considered in [17], where the authors seek to identify whether all spectrum bands provide the same performance. For example, low frequency spectrum, such as the $600 \mathrm{MHz}$ band considered in our paper, is generally viewed as more desirable than higher frequencies because of its propagation properties.

Scaling laws in wireless and wired networks have been studied in various contexts [18-21]. The work of Bolcskei et al., for example, focuses on the gains realized by increasing the number of antennas in a MIMO relay network. This work falls under the broad category of papers that analyze the dimensioning of telecommunications networks. Such papers are crucial in providing a better understanding of the relationship between resource allocation and system performance, allowing policy makers to look past the current state of the market.

Finally, spectrum markets have been the subject of many papers in recent years. For instance, the work in [22] analyzes the outcome of a game-theoretic pricing competition between providers in private commons. In the works by Jagannathan et al. [23], Kasbekar and Sarkar [24], Duan et al. [25], Niyato and Hossain [26], Sengupta and Chatterjee [27] and Xing et al. [28], game theoretic approaches to spectrum auctioning and leasing are analyzed. 


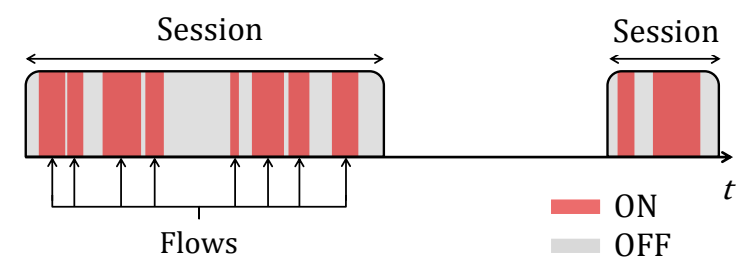

Fig. 1. Illustration of sessions and flows. Each session consists of one or more flows separated by idle periods.

None of the previous work surveyed here considers the impact of spectrum reservation for smaller providers on the competitiveness of a wireless market. The identification of the optimal carrier aggregation and the scaling laws provided thereunto, as well as simple methods of calculating it, are the unique contributions of this paper.

\section{MODEL}

In this section, we introduce the network model considered and the accompanying notation. We consider a small provider with a finite capacity $C>1$, which consists of the number of carriers in the spectrum owned by the provider. For example, in an LTE network configuration, these carriers could be interpreted as the resource blocks.

To realistically model network traffic, such as web browsing and streaming applications, we assume that the user demand consists of a sequence of session arrivals that follow a Poisson process with rate $\lambda>0$ [29]. A session consists of a combination of arbitrarily distributed and possibly correlated flows, generated by the same user or application. Each session consists of "on" and "off" periods within, where an "on" period means that a flow is generating traffic. Figure 1 provides an illustration of sessions and flows. Without loss of generality, we assume that the total "on" time within an individual session follows a general probability distribution and has a mean equal to one, independently of other requests and arrival times. Each flow has a peak rate that corresponds to the capacity of a carrier. If an arriving flow finds all the carriers busy, it is lost, but the rest of the session proceeds as normal. Note that standard voice calls are a special case of this model, for which a session consists of a single flow.

Under the above statistical assumptions, the probability that a flow is lost (blocked), is given by the Erlang-B formula [30]:

$$
E(\lambda, C)=\frac{\lambda^{C} / C !}{\sum_{k=0}^{C} \lambda^{k} / k !} .
$$

The above formula is insensitive to all traffic characteristics, except for the mean number of session arrivals per time unit $\lambda$.
The provider finds itself in the same competitive spectrum market as a larger network provider that has similar network parameters, but scaled by a multiplicative factor $n>1$ (i.e., its session arrival rate is $\lambda n$ and capacity $C n$ ). We refer to the parameter $n$ as the scaling factor.

The objective of the smaller provider is to meet the quality of service (QoS) of the larger provider, given by its Erlang blocking probability formula. This can be achieved through making use of the spectrum set aside and implementing carrier aggregation. Our goal is to identify the optimal level of carrier aggregation and investigate how it changes with the network parameters $\lambda$ and $C$ and the scaling factor $n$.

\section{Quality-Driven APPROXIMATION OF ERLANG-B FORMULA}

The Erlang-B formula given by Eq. (1) does not easily yield itself to analysis due to the summand and the factorial functions. Therefore, we seek an approximation of the Erlang-B formula that is more tractable. One such approximation is obtained through the consideration of a quality driven (QD) regime, characterized by $C \rightarrow \infty$, $\lambda \rightarrow \infty$ and the following relationship:

$$
C=\lambda(1+\gamma)
$$

where $\gamma>0$ is a constant representing the service grade. In a QD regime, the provider positions itself in terms of capacity with respect to its load so that it offers a high quality service (e.g., low probability of blocking or waiting).

The approximation that we will obtain under the QD regime works well for large values of $C$. Moreover, the approximation is asymptotically exact since the underlying stochastic process, when properly normalized, weakly converges to an Ornstein-Uhlenbeck diffusion process as $C \rightarrow \infty$ [12]. Before we establish the QD approximation to the Erlang-B formula, it is beneficial to recall the following fundamental inequality of the logarithm function:

$$
x-1 \geq \ln (x) \geq 1-\frac{1}{x}, \quad x>0,
$$

which we can rewrite as:

$$
x \geq \ln (1+x) \geq \frac{x}{1+x}, \quad x>0 .
$$

Under the QD regime we propose the following asymptotically exact approximation to the Erlang-B, which we will use in the rest of the paper:

Lemma IV.1 Under a $Q D$ regime such that $C=\lambda(1+$ $\gamma)$, the Erlang-B formula satisfies:

$$
\lim _{\lambda \rightarrow \infty} \frac{E(\lambda, C)}{\left(\sqrt{2 \Pi C}(1+\gamma)^{C} e^{-\lambda \gamma}\right)^{-1}}=1 .
$$


Proof. We first establish a relationship between the delay probability formula (Erlang-C) given by:

$$
E_{c}(\lambda, C)=\frac{\frac{\lambda^{C}}{C !} \frac{C}{C-\lambda}}{\sum_{k=0}^{C-1} \frac{\lambda^{k}}{k !}+\frac{\lambda^{C}}{C !} \frac{C}{C-\lambda}},
$$

and the Erlang-B formula. From the relationship between Erlang-B and Erlang-C provided in [31], it can be shown that:

$$
E(\lambda, C)=\frac{(1-\rho) E_{c}(\lambda, C)}{1-\rho E_{c}(\lambda, C)},
$$

where $\rho=\lambda / C=\frac{1}{1+\gamma}$ in a QD regime. Using the results provided in Section 16 of [14] for the analysis of queuing systems in the QD regime we obtain:

$$
E_{c}(\lambda, C)=\frac{e^{\lambda \gamma}+o(1 / \lambda)}{\sqrt{2 \Pi C} \gamma(1+\gamma)^{C-1}+e^{\lambda \gamma}+o(1 / \lambda)} .
$$

Substituting Eq. (5) for $E_{c}(\lambda, C)$ and Eq. (2) for $C$ into Eq. (4) we get:

$$
E(\lambda, C)=\frac{1+o(1 / \lambda)}{g(\lambda, \gamma)+1+o(1 / \lambda)},
$$

where $g(\lambda, \gamma)=\sqrt{2 \pi \lambda(1+\gamma)}\left((1+\gamma)^{(1+\gamma)} e^{-\gamma}\right)^{\lambda}$.

Now we will show that $g(\lambda, \gamma)$ is the dominating term in the denominator as $\lambda$ gets large. Observe that $(1+$ $\gamma)^{(1+\gamma)} \geq e^{\gamma}$ since taking the natural log of both sides we obtain:

$$
\begin{gathered}
(1+\gamma) \ln (1+\gamma) \geq \gamma \\
\ln (1+\gamma) \geq \frac{\gamma}{1+\gamma},
\end{gathered}
$$

which we know to be true from Eq. (3). Therefore $g(\lambda, \gamma)$ gets arbitrarily large with $\lambda$. We conclude that:

$$
\lim _{\lambda \rightarrow \infty} \frac{1}{g(\lambda, \gamma)}=0
$$

Hence:

$$
\lim _{\lambda \rightarrow \infty} \frac{E(\lambda, C)}{g(\lambda, \gamma)^{-1}}=\lim _{\lambda \rightarrow \infty} \frac{\frac{1+o(1 / \lambda)}{g(\lambda, \gamma)+1+o(1 / \lambda)}}{(g(\lambda, \gamma))^{-1}}=1 .
$$

Finally, we obtain $g(\lambda, \gamma)=\sqrt{2 \pi C}(1+\gamma)^{C} e^{-\lambda \gamma}$ through Eq. (2).

Lemma IV.1 states that the Erlang-B formula can be approximated by (and is asymptotically equal to) the following expression, which we refer to as the $Q D$ formula:

$$
E(\lambda, C) \simeq\left(\sqrt{2 \pi C}(1+\gamma)^{C} e^{-\lambda \gamma}\right)^{-1} .
$$

Figure 2 compares the Erlang-B and QD formulas, for carrier capacities typical to an LTE network [32]. Clearly, the values obtained are almost indistinguishable.

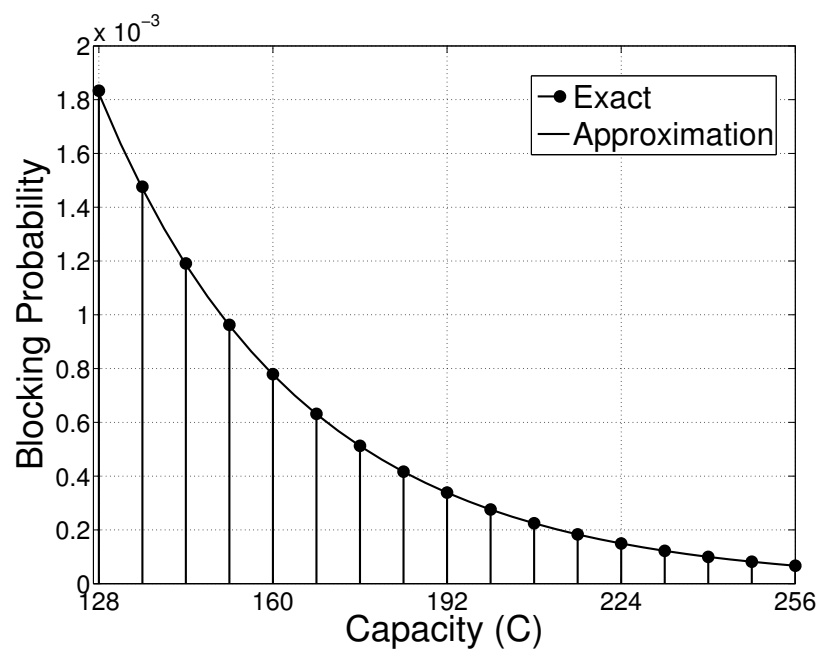

Fig. 2. QD Approximation with $C=(1+\gamma) \lambda$ and $\gamma=0.25$. The stem plot is the Erlang-B formula given by Eq. (1) while the line plot is the QD formula given by Eq. (6) .

All of the results presented in the rest of this paper are based the QD formula. Numerical examples will be provided to confirm their accuracy.

\section{MAin Results}

\section{A. Optimal Carrier Aggregation}

In this subsection, we define the problem of optimal carrier aggregation and provide numerical methods on calculating the level needed. Smith and Whitt [33] show that the Erlang-B formula is upwards scalable, that is:

$$
E(\lambda, C)>E(\lambda n, C n) .
$$

Thus, flows in a larger network experience a smaller blocking probability than that in a smaller network operating under a similar traffic load $\rho=\lambda / C$. This result is not surprising to teletraffic engineers, who know that combining two networks into a larger network results in better performance due to statistical multiplexing.

Therefore, when two providers experience similar loads but differ in network sizes in terms of the number of carriers they each possess, the larger provider initially provides an improved service to its users. Hence the smaller provider is inherently at a disadvantage in a competitive spectrum market.

We now turn our attention to the possibility of the smaller provider increasing its capacity by carrier aggregation. This way, the smaller provider can decrease the blocking probability experienced by its users. Let $\psi^{*}(n)$ denote the minimum (optimal) level of carrier aggregation the smaller provider needs to increase its network capacity to a size that achieves the same 


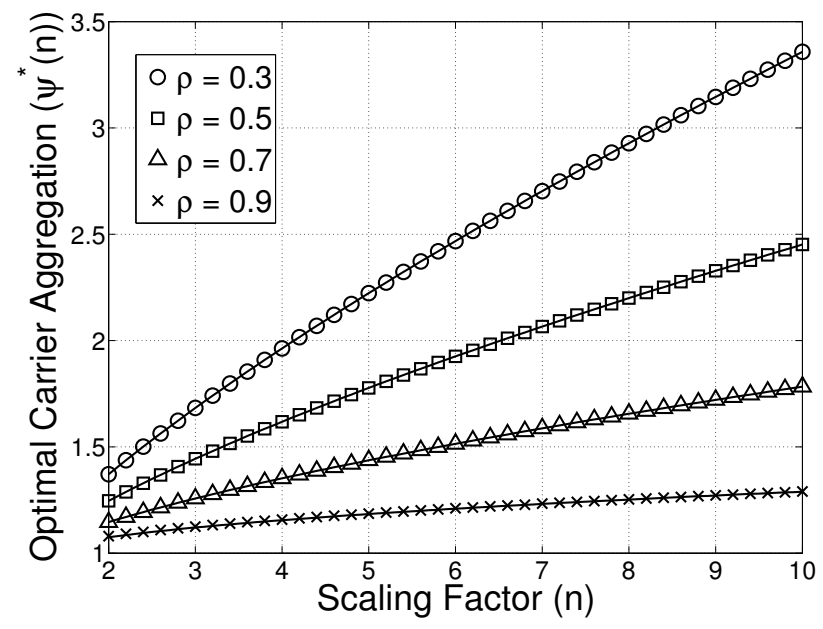

Fig. 3. Optimal level of carrier aggregation $\psi^{*}(n)$ of the smaller provider with respect to scaling factor $n$ for different initial traffic loads $\rho=\lambda / C$. Solid lines are exact, markers are QD approximation, and $C=50$.

blocking performance as the larger provider, namely $E\left(\lambda, C \psi^{*}(n)\right)=E(\lambda n, C n)^{1}$. Formally:

$$
\psi^{*}(n) \triangleq \min \{\psi: E(\lambda, C \psi) \leq E(\lambda n, C n)\}
$$

Using the QD formula given by Eq. (6), we get:

$$
\begin{aligned}
E(\lambda, C \psi) & \simeq\left(\sqrt{2 \pi C \psi}\left(1+\gamma^{\prime}\right)^{C \psi} e^{-\lambda \gamma^{\prime}}\right)^{-1}, \\
E(\lambda n, C n) & \simeq\left(\sqrt{2 \pi C n}(1+\gamma)^{C n} e^{-\lambda n \gamma}\right)^{-1}
\end{aligned}
$$

where $C \psi=\lambda\left(1+\gamma^{\prime}\right)$ and hence $\left(1+\gamma^{\prime}\right)=\psi(1+\gamma)$. Then we can rewrite Eq. (8) as:

$\psi^{*}(n) \triangleq \min \left\{\psi: \sqrt{\frac{\psi}{n}}\left(\frac{(1+\gamma)^{C}}{e^{\lambda \gamma}}\right)^{\psi-n} \frac{\psi^{C \psi}}{e^{\lambda(\psi-1)}} \geq 1\right\}$

As the left hand side of the inequality in Eq. (11) is increasing in $\psi$, equivalently $\psi^{*}(n)$ is the solution of:

$$
\sqrt{\frac{\psi}{n}}\left(\frac{(1+\gamma)^{C}}{e^{\lambda \gamma}}\right)^{\psi-n} e^{\lambda}\left(\frac{\psi^{C}}{e^{\lambda}}\right)^{\psi}=1
$$

Eq. (12) provides a fast way of numerically calculating the optimal level of carrier aggregation needed, which can be achieved using a binary search procedure as the left hand side is increasing in $\psi$. In Figure 3 we illustrate the calculated values of the optimal level of carrier aggregation using the QD formula and the exact Erlang-B formula. One can observe that the calculations

\footnotetext{
${ }^{1}$ While $C \psi$ must be an integer value when using Eq. (1), there exis continuous relaxations of the Erlang-B formula [34]. Furthermore, as the capacity tends to infinity in a QD regime, $\psi$ can be treated as continuous.
}

based on the QD formula work well: even at a capacity as low as 50 carriers, the maximum percentage error ${ }^{2}$ between the QD approximation and the actual Erlang-B calculation is $0.5714 \%$, which occurs when $\rho=0.9$.

\section{B. Structural Properties of Optimal Carrier Aggregation}

In this section, we derive structural properties of optimal carrier aggregation. Specifically, we analyze the asymptotic behavior of the optimal carrier aggregation with respect to the scaling factor $n$. We also show that the amount of carrier aggregation needed diminishes when the initial traffic load at which the providers operate is higher.

1) Scaling Laws: From Eq. (7), it follows that the difference between the blocking probabilities of the two providers increases with the scaling factor $n$. Thus the disadvantaged provider needs to aggregate more carriers as $n$ gets larger. We next provide asymptotic lower and upper bounds on the optimal level of carrier aggregation as a function of the scaling factor $n$ :

Theorem V.1 (Capacity Scaling Law) Consider two providers differing by a scaling factor of $n$. Then the optimal level of carrier aggregation with respect to the scaling factor $n$ satisfies:

$$
\begin{aligned}
& \text { 1) } \psi^{*}(n)=o\left(\frac{n}{\log (n)}\right) \\
& \text { 2) } \psi^{*}(n)=\omega\left(n^{\alpha}\right), \text { for any constant } \alpha<1,
\end{aligned}
$$

where $o(\cdot)$ and $\omega(\cdot)$ are standard asymptotic notations respectively representing strict upper and lower asymptotic limiting behavior of the functions within the parentheses.

Proof of Theorem. Making use of Eq. (2), let us rearrange Eq. (12) to obtain:

$$
\sqrt{\psi} a^{\lambda \psi} \psi^{\lambda(1+\gamma) \psi}=\sqrt{n} a^{\lambda n} e^{\lambda(\psi-1)},
$$

where $a=\frac{(1+\gamma)^{(1+\gamma)}}{e^{\gamma}}$. Taking the $\log _{a}(\cdot)$ (which we will simply denote by $\log (\cdot)$ to alleviate the notation) of both sides and dividing by $\lambda$ yields:

$$
\begin{aligned}
\frac{\log (\psi)}{2 \lambda}+(1+\gamma) & \psi \log (\psi)+\psi \\
& =\frac{\log (n)}{2 \lambda}+n+(\psi-1) \log (e)
\end{aligned}
$$

We will prove the upper and lower bounds separately.

1. Let us assume that $\psi=\frac{n}{\log _{a}(n)}$. We will check the upper bound by substituting for $\psi$ in Eq. (13) and

$$
\begin{aligned}
& { }^{2} \text { Calculated by }\left|\frac{\psi^{Q D}-\psi^{\text {Erlang }}}{\psi^{\text {Erlang }}}\right| \cdot 100 \text {, where } \psi^{Q D} \text { is given by } \\
& \text { Eq. (6) and } \psi^{\text {Erlang }} \text { is given by Eq. (1). }
\end{aligned}
$$


showing that as $n \rightarrow \infty$, the left hand side is strictly greater than the right hand side. We get:

$$
\begin{aligned}
\frac{1}{2 \lambda} \log \left(\frac{n}{\log (n)}\right)+\frac{n(1+\gamma)}{\log (n)} \log \left(\frac{n}{\log (n)}\right)+ \\
\frac{n}{\log (n)}>\frac{\log (n)}{2 \lambda}+n+\frac{n \log (e)}{\log (n)}-\log (e) .
\end{aligned}
$$

Canceling the common terms and rearranging, we can rewrite this relationship as:

$$
\begin{aligned}
\gamma \log (n)+1 & +\frac{\log (e) \log (n)}{n}> \\
& \left(1+\gamma+\frac{\log (n)}{2 \lambda n}\right) \log (\log (n))+\log (e),
\end{aligned}
$$

which is true for sufficiently large $n($ as $\log (n)=o(n))$. Thus, we have demonstrated that when $\psi=\frac{n}{\log (n)}$ and $n$ is sufficiently large, the left hand side of Eq. (12) is strictly greater than one. Since the left hand side of (12) is increasing in $\psi$, we conclude that there must be some $\psi^{\prime}<\psi=\frac{n}{\log (n)}$ that satisfies Eq. (12).

2. Assume that $\psi=n^{\alpha}, \alpha<1$. We will demonstrate that substituting for $\psi$ in Eq. (13) results in the left hand side being strictly smaller than the right hand side as $n \rightarrow \infty$. We get:

$$
\begin{aligned}
& \frac{\alpha \log (n)}{2 \lambda}+\alpha(1+\gamma) n^{\alpha} \log (n)+n^{\alpha}< \\
& n+\frac{\log (n)}{2 \lambda}+\log (e)\left(n^{\alpha}-1\right) .
\end{aligned}
$$

Dividing by $n^{\alpha}$ and collecting and rearranging the terms we have:

$$
\begin{aligned}
\alpha(1+\gamma) \log (n)+1+ & \frac{\log (e)}{n^{\alpha}}< \\
& n^{1-\alpha}+\frac{(1-\alpha) \log (n)}{2 \lambda n^{\alpha}}+\log (e),
\end{aligned}
$$

which, since $n=\omega(\log (n))$, holds as $n$ gets large. Therefore, when $\psi=n^{\alpha}, \alpha<1$, the left hand side of Eq. (12) is smaller than one. Hence, there must be another $\psi^{\prime}>\psi=n^{\alpha}$ that satisfies Eq. (12).

Theorem V.1 states that $n / \log (n)$ and $n^{\alpha}$ are asymptotic upper and lower bounds on $\psi^{*}(n)$ respectively. Therefore as the scaling factor increases, the level of optimal carrier aggregation scales sub-linearly but also asymptotically approaches (though never achieves) a linear relationship. This behavior can be observed in Figure 3.

2) Traffic Load: Having provided scaling laws on optimal carrier aggregation with respect to the scaling factor $n$, we now turn our attention to the scaling with respect to the traffic load.

In the next theorem, we state that the optimal level of carrier aggregation needed by the smaller provider is lower in a market where both providers experience a high initial traffic load. Therefore, in high load markets it is easier for a smaller provider to aggregate spectrum in order to compete.

Theorem V.2 (Traffic Load Scaling Law) Let $\rho_{j}$ denote the traffic load in a market $j$, which consist of two providers that differ in size by a scale of $n$ such that:

$$
\rho_{j}=\frac{\lambda_{j} n}{C n}=\frac{\lambda_{j}}{C} \text { for } j=1,2 .
$$

Further define $\psi_{j}^{*}(n)$ to be the optimal level of carrier aggregation for the smaller provider in the market characterized by load $\rho_{j}$. Then, for two given traffic loads, such that $\rho_{1}>\rho_{2}$,

$$
\psi_{1}^{*}(n)<\psi_{2}^{*}(n) .
$$

The next two lemmas, whose proofs are provided in the technical report [35], give inequalities that we will use in the proof of our theorem.

Lemma V.1 For $\gamma>0$ and $n>1$ :

$$
(1+n \gamma) \ln (1+n \gamma)>n(1+\gamma) \ln (1+\gamma) .
$$

Lemma V.2 For $\gamma>0$ and $n \geq 1$ :

$$
\psi^{*}(n)<\rho+(1-\rho) n .
$$

Proof of Theorem. Assume that the different loads are caused by different arrival rates such that $\lambda_{1}>\lambda_{2}$ while the capacity is kept constant at $C$. Then we have:

$$
C=\lambda_{1}\left(1+\gamma_{1}\right)=\lambda_{2}\left(1+\gamma_{2}\right) \text {. }
$$

It immediately follows that $\left(1+\gamma_{1}\right)<\left(1+\gamma_{2}\right)$. Using Eq. (12), the following need to be satisfied in optimality:

$$
\begin{aligned}
& \sqrt{\frac{\psi}{n}}\left(\frac{\left(1+\gamma_{1}\right)^{C}}{e^{\lambda_{1} \gamma_{1}}}\right)^{\psi-n} \frac{\psi^{C \psi}}{e^{\lambda_{1}(\psi-1)}}=1, \\
& \sqrt{\frac{\psi}{n}}\left(\frac{\left(1+\gamma_{2}\right)^{C}}{e^{\lambda_{2} \gamma_{2}}}\right)^{\psi-n} \frac{\psi^{C \psi}}{e^{\lambda_{2}(\psi-1)}}=1 .
\end{aligned}
$$

Suppose $\psi_{2}^{*}(n)=\psi$ and satisfies Eq. (16). Then we need to show that the left hand side of Eq. (15) is strictly greater than one when $\psi_{1}^{*}(n)=\psi$.

Let us rewrite the left hand side of Eq. (16) as the following:

$$
\begin{aligned}
& \sqrt{\frac{\psi}{n}}\left(\frac{\left(1+\gamma_{2}\right)^{C}}{e^{\lambda_{2} \gamma_{2}}}\right)^{\psi-n} \frac{\psi^{C \psi}}{e^{\lambda_{2}(\psi-1)}}= \\
& \quad \sqrt{\frac{\psi}{n}}\left(\frac{\left(1+\gamma_{1}\right)^{C}}{e^{\lambda_{1} \gamma_{1}}}\right)^{\psi-n} \frac{\psi^{C \psi}}{e^{\lambda_{1}(\psi-1)}} \frac{\left(\lambda_{1} / \lambda_{2}\right)^{C(\psi-n)}}{e^{\left(\lambda_{1}-\lambda_{2}\right)(1-n)}} .
\end{aligned}
$$

Now we will demonstrate that:

$$
e^{\left(\lambda_{1}-\lambda_{2}\right)(n-1)}<\left(\lambda_{1} / \lambda_{2}\right)^{C(n-\psi)} .
$$


Start by taking the $\ln (\cdot)$ of both sides of (17) to get:

$$
\left(\lambda_{1}-\lambda_{2}\right)(n-1)<C(n-\psi) \ln \left(\lambda_{1} / \lambda_{2}\right) .
$$

Since $\ln \left(\lambda_{1} / \lambda_{2}\right) \geq \frac{\lambda_{1}-\lambda_{2}}{\lambda_{1}}$ by inequality (3) and $C=$ $\lambda_{1}\left(1+\gamma_{1}\right)$ :

$$
C(n-\psi) \ln \left(\lambda_{1} / \lambda_{2}\right) \geq\left(1+\gamma_{1}\right)(n-\psi)\left(\lambda_{1}-\lambda_{2}\right) .
$$

From Lemma V.2 we have that $\psi<\rho+(1-\rho) n$, which by substituting $\rho=1 /\left(1+\gamma_{1}\right)$ and rearranging the terms, can be rewritten as:

$$
\left(1+\gamma_{1}\right)(n-\psi)>(n-1) .
$$

Combining Eqs. (18) and (19), we get to the inequality in (17). Then we can claim that:

$$
\begin{aligned}
& \sqrt{\frac{\psi}{n}}\left(\frac{\left(1+\gamma_{1}\right)^{C}}{e^{\lambda_{1} \gamma_{1}}}\right) \psi-n \frac{\psi^{C \psi}}{e^{\lambda_{1}(\psi-1)}} \\
& \quad>\sqrt{\frac{\psi}{n}}\left(\frac{\left(1+\gamma_{2}\right)^{C}}{e^{\lambda_{2} \gamma_{2}}}\right)^{\psi-n} \frac{\psi^{C \psi}}{e^{\lambda_{2}(\psi-1)}}=1 .
\end{aligned}
$$

Therefore, by continuity and the fact that the left hand side of (15) is increasing in $\psi$, there must be another

$$
\psi^{\prime}<\psi=\psi_{2}^{*}(n)
$$

that satisfies Eq. (15). Hence, $\psi_{1}^{*}(n)<\psi_{2}^{*}(n)$.

Theorem V.2 states that the level of carrier aggregation needed to provide a service level that can compete with the larger provider in the market is higher (lower) under a low (high) traffic load, which is also illustrated in Figure 3. This implies that the marginal benefit of aggregating spectrum is higher when the providers are operating under a higher load.

\section{General Bounds}

In this section, we seek to establish an upper bound that holds for all possible values of the scaling factor $n$. We will first establish that optimal carrier aggregation $\psi^{*}(n)$ is concave in $n$, the proof for which can be found in the technical report [35]:

Lemma V.3 (Concavity) For $1 \leq n_{1}<n_{2}$,

$$
\psi^{\prime *}\left(n_{1}\right)<\psi^{\prime *}\left(n_{2}\right) .
$$

Given the derivative of $\psi^{*}(n)$ is decreasing in $n$, we next establish the tightest possible linear upper bound on $\psi^{*}(n)$ :

Theorem V.3 (Linear Upper Bound) For $\gamma>0$ and $n \geq 1$ :

$$
\psi^{*}(n) \leq(1-f(\rho))+f(\rho) n,
$$

where $f(\rho)=1-\frac{1-\rho}{\frac{1}{2 C}+\ln (1 / \rho)}$.

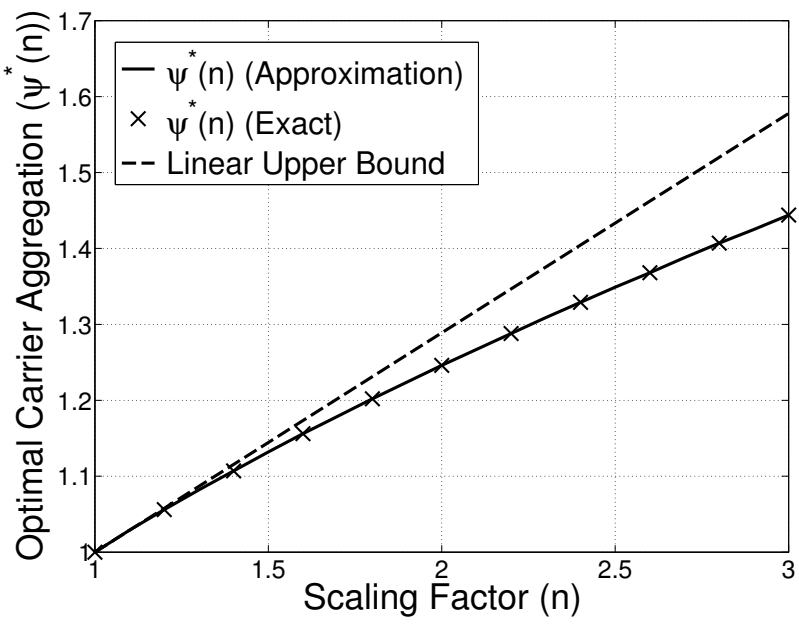

Fig. 4. Linear upper bound on the optimal level of carrier aggregation $\psi^{*}(n)$ provided in Theorem V.3, with $C=50$ and $\rho=0.5$.

Proof of Theorem. We will start our proof by providing a linear function of the form $g(n)=a+b n$, where $a$ and $b$ are constants, that is equal to $\psi^{*}(n)$ when $n=1$ and has the same derivative at that point. From the way we defined $\psi^{*}(n)$ in Eq. (8), it follows that $\psi^{*}(1)=1$. Then $g(n)=(1-b)+b n$ in order to satisfy this inequality.

Taking the derivative of the both sides of Eq. (12) and rearranging the terms one can obtain the following:

$$
\psi^{\prime *}(n)=\frac{\frac{1}{2 n}+C \ln (1+\gamma)-\lambda \gamma}{\frac{1}{2 \psi^{*}(n)}+C \ln (1+\gamma)+C \ln \left(\psi^{*}(n)\right)} .
$$

Note that Eq. (21) depends on the exact value of $\psi^{*}(n)$. Evaluating this expression at $\psi^{*}(1)=1$ yields:

$$
\left.\frac{d \psi^{*}(n)}{d n}\right|_{n=1, \psi^{*}(1)=1}=\frac{\frac{1}{2}+C \ln (1+\gamma)-\lambda \gamma}{\frac{1}{2}+C \ln (1+\gamma)} .
$$

Rearranging the terms in Eq. (22) and substituting $\rho$ for $\frac{1}{1+\gamma}$, we obtain:

$$
\left.\frac{d \psi^{*}(n)}{d n}\right|_{n=1, \psi^{*}(1)=1}=1-\frac{1-\rho}{\frac{1}{2 C}+\ln (1 / \rho)} .
$$

Then $b=1-\frac{1-\rho}{\frac{1}{2 C}+\ln (1 / \rho)}$ and

$$
g(n)=\frac{1-\rho}{\frac{1}{2 C}+\ln (1 / \rho)}+\left(1-\frac{1-\rho}{\frac{1}{2 C}+\ln (1 / \rho)}\right) n
$$

Now we will show that $g(n) \geq \psi^{*}(n)$ for $n \geq 1$. Observe that $g(1)=\psi^{*}(1)$. In Lemma V.3 we have established that the derivative of optimal carrier aggregation with respect to the scaling factor $n$ is decreasing in $n$. Then we can state that

$$
\frac{d g(n)}{d n} \geq \frac{d \psi^{*}(n)}{d n} \text { for any } n \geq 1 \text {. }
$$


Let $h(n)=g(n)-\psi^{*}(n)$. Taking the derivative with respect to $n$ we get:

$$
\frac{d h(n)}{d n}=\frac{d g(n)}{d n}-\frac{d \psi^{*}(n)}{d n} \geq 0 .
$$

By mean value theorem there exists an $n_{0}$ such that:

$$
\frac{d h\left(n_{0}\right)}{d n}=\frac{h(n)-h(1)}{n-1}=\frac{g(n)-\psi^{*}(n)}{n-1} \geq 0 .
$$

Since $n \geq 1$ we conclude that $g(n) \geq \psi^{*}(n)$.

Theorem V.3 provides a way to quickly calculate an upper bound on the optimal carrier aggregation, which is rather tight for small values of the scaling factor $n$ as illustrated in Figure 4. However, since $\psi^{*}(n)$ is concave, as the scaling factor increases, the linear upper bound diverges from the actual value. The strength of the linear upper bound that we provide lies in its ability to provide simple insight on the impact of network parameters on optimal carrier aggregation.

As a possible solution to the divergence of the linear upper bound, one could seek to obtain a piece-wise linear upper bound expression on $\psi^{*}(n)$ by using the results provided in Lemma V.3 and Theorem V.3. Starting at $\psi^{*}(1)=1$, one can use the linear bound provided in Eq. (20) to approximate the value of $\psi^{*}(n)$ at a larger value of $n$, which can then be used to obtain the derivative $\psi^{\prime}(n)$ provided in Eq. (21). The derivative value can then be assumed to be the linear slope of $\psi^{*}(n)$, and the calculation procedure starts over.

Next, we propose a simple algorithmic procedure to compute a piece-wise linear bound on $\psi^{*}(n)$ (see Algorithm 1). The algorithm takes as input the scaling factor $n$, referred to as Scale, as well as the step size, referred to as StepSize, that defines the distance between points at which the slope of the bound is recalculated. The procedure starts from the known point of $\psi^{*}(1)=1$ and uses the linear bound established to calculate the bound on $\psi^{*}$ at every evaluation point determined by the step size until the target scaling factor is reached.

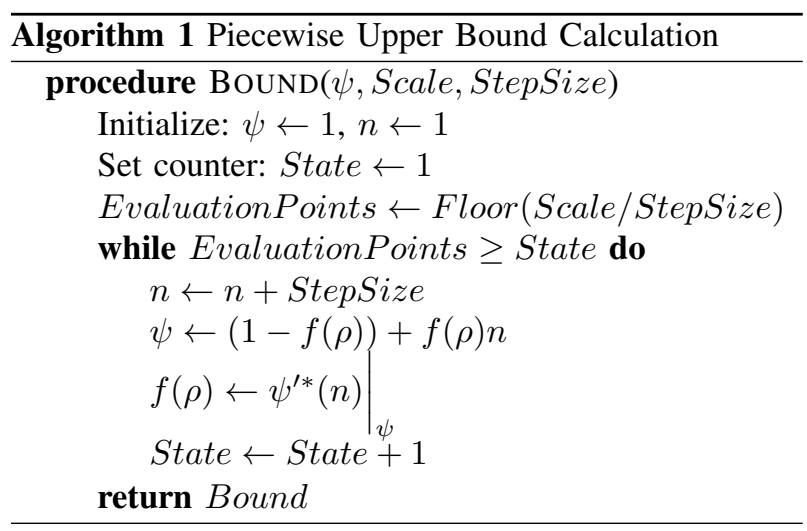

Using Algorithm 1, if the step size is chosen small enough, the bound on $\psi^{*}(n)$ will approach the real value. Therefore, one can obtain a relatively tight piecewise linear upper bound on $\psi^{*}(n)$, which is illustrated in Figure 5 for several different traffic loads, with a step size of 1 .

\section{Numerical Examples}

In this section, we provide numerical examples, where we calculate how much spectrum needs to be aggregated to preserve competition in different markets.

Consider two providers in a spectrum market with network parameters given as follows:

$$
\left(\lambda_{1}, C_{1}\right)=(90,150) \text { and }\left(\lambda_{2}, C_{2}\right)=(60,100) .
$$

The capacity numbers provided here are in line with the spectrum holdings of Verizon and T-Mobile in the New York City area, which respectively are $30 \mathrm{MHz}$ and $20 \mathrm{MHz}$ (translated into the number of resource blocks from Table I), according to the FCC's Spectrum Dashboard [36]. In this example, the scaling factor is $n=150 / 100=1.5$ and both providers are in a moderately loaded market with $\rho=90 / 150=60 / 100=0.60$. Using Equation (12), we obtain the carrier aggregation needed by the smaller provider: $\psi^{*}(1.5)=1.102$.

This result tells us that in order to provide the same level of service as the larger provider, the smaller provider needs to increase its capacity at least by 1.102 times its current value. Therefore, $\lceil 100 \times 0.102\rceil=$ 11 additional carriers are needed to bring the smaller provider's service level in line with that of the larger provider. Taking a single carrier to be a resource block in an LTE deployment, the smallest LTE bandwidth that matches this requirement has a bandwidth of $3 \mathrm{MHz}$ from Table I. This is the amount of spectrum that the smaller provider needs to aggregate in order to guarantee its ability to compete with the larger provider.

Next, we consider two different markets: (i) a market where the spectrum holdings of the providers have the same scaling factor but the traffic load $\rho$ is higher and (ii) a market where there is an increase in the scaling factor $n$ while the traffic load $\rho$ is the same.

( $i$ ) Consider a market where the scaling factor is $n=$ 1.5 while the traffic load of the market increases to $\rho=$ 0.9 . The parameters of the two providers are now as follows:

$$
\left(\lambda_{1}, C_{1}\right)=(135,150) \text { and }\left(\lambda_{2}, C_{2}\right)=(90,100) .
$$

Under the new load, the carrier aggregation needed by the smaller provider becomes $\psi^{*}(1.5)=1.037$.

Thus, $\lceil 100 \times 0.037\rceil=4$ additional carriers are needed by the smaller provider, fewer than the number 

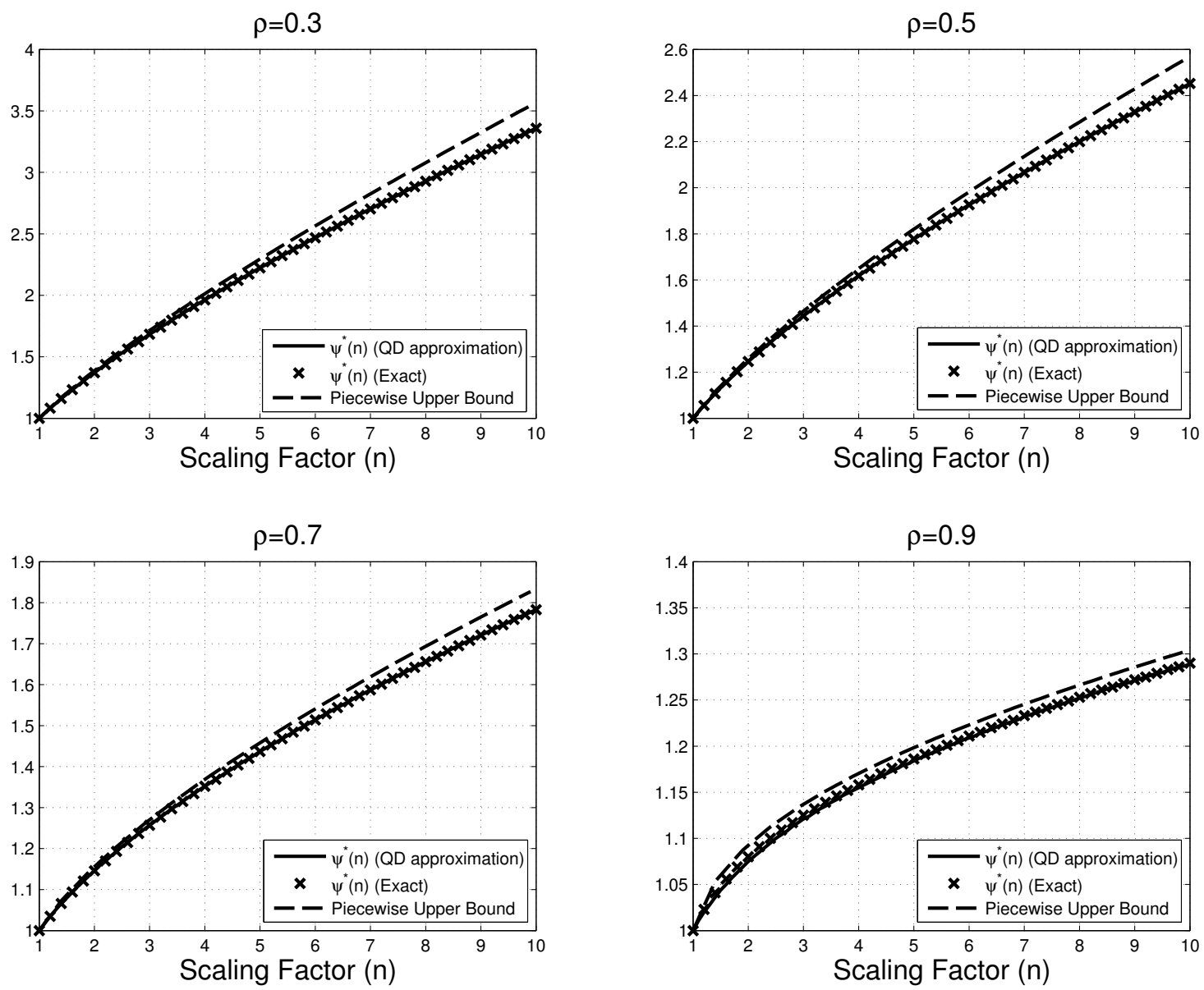

Fig. 5. $C=50$ - Piecewise linear upper bounds on the optimal level of carrier aggregation $\psi^{*}(n)$ obtained by the initial linear upper bound provided in Theorem V.3, the slope of which is then adjusted at integer values of the scaling factor $n$ using the derivative expression provided in Eq. (21).

\begin{tabular}{|c|c|c|c|c|c|c|}
\hline Bandwidth & $1.4 \mathrm{MHz}$ & $3 \mathrm{MHz}$ & $5 \mathrm{MHz}$ & $10 \mathrm{MHz}$ & $15 \mathrm{MHz}$ & $20 \mathrm{MHz}$ \\
\hline Resource Blocks & 6 & 15 & 25 & 50 & 75 & 100 \\
\hline
\end{tabular}

TABLE I

LTE BANDWIDTH CONFIGURATIONS AND ASSOCIATED NUMBER OF RESOURCE BLOCKS AS SPECIFIED IN 3GPP RELEASE 8 [10].

of carriers calculated before and in line with Theorem V.2. Under the same LTE scenario considered previously, Table I indicates that aggregating a minimum of $1.4 \mathrm{MHz}$ of spectrum in the market with a higher traffic load is enough to achieve the same goal.

(ii) This time, we consider a market where the scaling factor is increased to $n=6$ but the traffic load is the same as the first market (i.e., $\rho=0.6$ ). The parameters of the providers are given as follows:

$$
\left(\lambda_{1}, C_{1}\right)=(90,150) \text { and }\left(\lambda_{2}, C_{2}\right)=(15,25) .
$$

These numbers are in line with the spectrum holdings of Verizon and T-Mobile in Logan County, IL, which respectively are $30 \mathrm{MHz}$ and $5 \mathrm{MHz}$ (translated into the number of resource blocks from Table I), according the FCC's Spectrum Dashboard [36]. In this case, the carrier aggregation needed by the smaller provider is $\psi^{*}(6)=$ 1.719 .

This time, the smaller provider needs an additional $\lceil 100 \times 0.719\rceil=72$ carriers. Notice that the increase in the total capacity needed is smaller than the increase in the scaling factor since:

$$
\psi^{*}(6) / \psi^{*}(1.5)=1.559<6 / 1.5=4
$$

Under the same LTE scenario considered previously, Table I indicates that aggregating a minimum of $15 \mathrm{MHz}$ of spectrum is needed to achieve the same goal, as the scaling factor increases to 6 . 


\section{E. Extension to Delay Systems}

In Section IV, we presented a QD regime approximation of the Erlang-B formula through Lemma IV.1. The assumption was that if all the carriers are busy upon the arrival of a flow, then the flow is lost. This is referred to as a loss system.

Our results can easily be extended to a delay system. In such a system, all active flows share the entire network capacity. If the number of flows exceeds $C$, then the flows can still be transmitted but at a rate below their peak rate. In that case, the flows will experience congestion and additional delay. The probability that an arrival flow experiences congestion is given by the Erlang-C formula:

$$
E_{c}(\lambda, C)=\frac{\frac{\lambda^{C}}{C !} \frac{C}{C-\lambda}}{\sum_{k=0}^{C-1} \frac{\lambda^{k}}{k !}+\frac{\lambda^{C}}{C !} \frac{C}{C-\lambda}} .
$$

This equation holds for the same general traffic model as presented in Section III [37].

Using the results of [14] for the analysis of queuing systems in the QD regime we have:

$$
E_{c}(\lambda, C) \simeq\left(\sqrt{2 \pi C} \gamma(1+\gamma)^{C-1} e^{\lambda \gamma}\right)^{-1}
$$

Through following similar steps as in Section V-A and replacing the QD formula of Erlang-B with Eq. (23), it is possible to show that the optimal carrier aggregation in a delay system is given by:

$$
\begin{aligned}
& \psi_{c}^{*}(n)=\min \{\psi: \sqrt{\frac{\psi}{n}}\left(\frac{(1+\gamma)^{C}}{e^{\lambda \gamma}}\right)^{\psi-n} . \\
&\left.\frac{\psi^{C \psi}}{e^{\lambda(\psi-1)}}\left(1+\frac{\psi-1}{\psi \gamma}\right) \geq 1\right\} .
\end{aligned}
$$

As the left hand side of the inequality in Eq. (11) is increasing in $\psi$, equivalently $\psi_{c}^{*}(n)$ is the solution of:

$$
\sqrt{\frac{\psi}{n}}\left(\frac{(1+\gamma)^{C}}{e^{\lambda \gamma}}\right)^{\psi-n} \frac{\psi^{C \psi}}{e^{\lambda(\psi-1)}}\left(1+\frac{\psi-1}{\psi \gamma}\right)=1
$$

Note that Eq. (25) is the same as Eq. (12) except for the $1+\frac{\psi-1}{\psi \gamma}$ term at the end. Since $1+\frac{\psi-1}{\psi \gamma}>1$ for $\psi>1$, one quickly concludes that the left hand side of Eq. (25) is always strictly greater than the left hand side of Eq. (12). In other words, for the same system parameters, the level of optimal carrier aggregation under the Erlang-C delay model is always smaller than that under the Erlang-B loss model:

$$
\psi_{c}^{*}(n)<\psi^{*}(n)
$$

Figure 6 illustrates this relationship. By replacing Eq. (12) with Eq. (25) and following a similar analysis,



Fig. 6. Comparison of optimal carrier aggregation under loss and delay systems, with $C=50$ and $\rho=0.7$.

the same structural properties given in Theorems V.1 and V.2 also hold for the QD Erlang-C formula. To give an example, we revisit Theorem V.1 here:

Theorem V.4 (Erlang-C Capacity Scaling Law) Consider two providers differing by a scaling factor of $n$. Then the optimal level of carrier aggregation with respect to the scaling factor $n$ satisfies:

$$
\begin{aligned}
& \text { 1) } \psi_{c}^{*}(n)=o\left(\frac{n}{\log (n)}\right) \\
& \text { 2) } \psi_{c}^{*}(n)=\omega\left(n^{\alpha}\right), \text { for any } \alpha<1,
\end{aligned}
$$

where $o(\cdot)$ and $\omega(\cdot)$ respectively represent strict upper and lower asymptotic limiting behavior on the function within the parentheses.

Proof of Theorem. Let us rearrange Eq. (25) to obtain:

$$
\sqrt{\frac{\psi}{n}}\left(\frac{(1+\gamma)^{C}}{e^{\lambda \gamma}}\right)^{\psi-n} \frac{\psi^{C \psi}}{e^{\lambda(\psi-1)}}=\frac{\psi \gamma}{\psi(1+\gamma)-1} .
$$

We will prove that $n / \log (n)$ and $n^{\alpha}$ are still asymptotic upper and lower bounds by showing that the term $\frac{\psi \gamma}{\psi(1+\gamma)-1}$ converges to a constant and thus does not affect the asymptotic relationships.

1. Assume that $\psi=\frac{n}{\log (n)}$. Then replacing $\psi$ in the right hand side term of Eq. (26) we obtain:

$$
\frac{\psi \gamma}{\psi(1+\gamma)-1}=\frac{\frac{n \gamma}{\log (n)}}{\frac{n}{\log (n)}(\gamma+1)-1},
$$

and:

$$
\lim _{n \rightarrow \infty} \frac{\frac{n \gamma}{\log (n)}}{\frac{n}{\log (n)}(\gamma+1)-1}=\frac{\gamma}{1+\gamma}=(1-\rho) .
$$


2. This time, assume that $\psi=n^{\alpha}, \alpha<1$. Then replacing $\psi$ in the right hand side term of Eq. (26) we obtain:

$$
\frac{\psi \gamma}{\psi(1+\gamma)-1}=\frac{n^{\alpha} \gamma}{n^{\alpha}(1+\gamma)-1}
$$

and

$$
\lim _{n \rightarrow \infty} \frac{n^{\alpha} \gamma}{n^{\alpha}(1+\gamma)-1}=\frac{\gamma}{1+\gamma}=(1-\rho)
$$

\section{F. Applications to Secondary Spectrum Markets}

We next discuss how our results on carrier aggregation apply to pricing games in secondary spectrum markets. In previous work [38], we identified the minimum (break-even) price at which it is profitable for a provider to start admitting secondary users. The breakeven price $p^{B E}$ is directly linked to the Erlang-B formula:

$$
p^{B E}=K E(\lambda, C),
$$

where $\lambda$ is the session arrival rate of primary users, $C$ is the network capacity, and $K$ is the price paid by primary users per session. Strikingly, the break-even price is insensitive to the secondary demand.

The break-even price plays a critical role in determining the Nash equilibrium of a game where two providers compete in prices to attract secondary demand. Without loss of generality, suppose that the break-even price of provider 1 is lower than that of provider 2 . Then, according to Theorem V.I in [38], the competition results in a price war that is won by provider 1 (i.e., provider 1 captures the entire market). One concludes that the outcome of the pricing game is directly related to the break-even prices, which in turn relate to the ErlangB formula.

Hence, the level of optimal carrier aggregation acts as an identifier of necessary network provisioning to obtain a competitive price advantage in a secondary spectrum market. All of our previous results, such as the structural properties with respect to scaling factors and traffic loads and the established general bounds can be readily applied to the question of how to strategically implement carrier aggregation in a secondary spectrum market.

\section{CONCLUDING REMARKS}

We investigated the impact of reserving spectrum for smaller providers, by providing computational methods, scaling laws, and bounds on the optimal carrier aggregation. Under a QD regime, we derived an approximation of the Erlang-B formula. This approximation is highly accurate as long as the number of carriers is large enough (e.g., above 50) and the spectrum utilization does not approach $100 \%$ (e.g., $90 \%$ or below), an assumption that is consistent with measurement studies [39].

Using the QD formula, we investigated optimal carrier aggregation by proving two scaling laws: (i) with respect to the scaling factor $n$ and (ii) with respect to the traffic load. Specifically, we obtained sub-linear (though close to linear) asymptotic upper and lower bounds in the form $\psi^{*}(n)=o(n / \log (n))$ and $\psi^{*}(n)=\omega\left(n^{\alpha}\right)$ for any $\alpha<$ 1. Then, we demonstrated that if the traffic load under which each provider operates increases, then the level of carrier aggregation required is reduced. This result indicates that the marginal benefit of carrier aggregation in a heavily loaded network is higher than that in a lightly loaded network.

Next, we derived an upper bound on $\psi^{*}(n)$ that applies to any value of $n$ and is provably the tightest possible. This upper bound explicitly relates to the network parameters and can provide regulators and market players with useful guidelines. We also provided a method of improving it to a piece-wise linear bound by iteratively approximating $\psi^{\prime}(n)$.

We explained how the results derived for loss systems, based on the Erlang-B formula, extend to delay systems based on the Erlang-C formula. We proved that for the same network parameters, the optimal level of carrier aggregation in a delay system is always smaller than in a loss system. Finally, we provided a relationship between the profitable pricing of users in secondary spectrum markets and the Erlang-B formula for which our results apply. Hence, the results on optimal carrier aggregation presented in this paper are directly applicable to pricing strategies in secondary spectrum markets, where providers can aggregate spectrum to lower their prices in a possible price war.

This paper focused on a single small provider implementing carrier aggregation. If several small providers are present in the same market, similar conclusions hold if the total carrier aggregation needed stays below the amount of spectrum reserved by the regulator. If the total amount of spectrum needed by all of the small providers exceeds the reserved amount, then one can expect competition between the small providers and possibly formation of coalitions. While beyond the scope of this paper, evaluating the impact of carrier aggregation on a market with multiple providers of different sizes is an interesting area left for future research.

Acknowledgments: This work was supported, in part, by the NSF grants CCF-0964652 and CNS-1117160.

\section{REFERENCES}

[1] "FCC adopts revised mobile spectrum holdings policies to preserve \& promote a competitive wireless market- 
place,” http://transition.fcc.gov/Daily_Releases/Daily_Business/ 2014/db0515/DOC-327109A1.pdf, The Federal Communications Commission, 2014.

[2] "Mobile spectrum holdings report and order" http://www.fcc. gov/document/mobile-spectrum-holdings-report-and-order, The Federal Communications Commission, 2014.

[3] "Verizon, AT\&T may face bidding limits in spectrum auction," http://online.wsj.com/news/articles/ SB10001424052702304626304579510154106120342, The Wall Street Journal, 2014.

[4] "FCC votes to restrict AT\&T, Verizon in 2015 spectrum auction," http://www.reuters.com/article/2014/05/15/us-usawireless-fcc-idUSBREA4E0RF20140515, Reuters, 2014.

[5] "FCC should create a $40 \mathrm{mhz}$ reserve for $600 \mathrm{mhz}$ auction, public interest groups urge," http://www.fiercewireless.com/story/fccshould-create-40-mhz-reserve-600-mhz-auction-public-interestgroups-urg/2015-02-25, Fierce Wireless, 2015.

[6] M. Iwamura, K. Etemad, M.-H. Fong, R. Nory, and R. Love, "Carrier aggregation framework in 3GPP LTEAdvanced [WiMAX/LTE update]," Communications Magazine, IEEE, vol. 48, no. 8, pp. 60-67, August 2010.

[7] W. Wang, Z. Zhang, and A. Huang, "Spectrum aggregation: Overview and challenges." Network Protocols \& Algorithms, vol. 2, no. 1, 2010.

[8] G. Yuan, X. Zhang, W. Wang, and Y. Yang, "Carrier aggregation for LTE-advanced mobile communication systems," Communications Magazine, IEEE, vol. 48, no. 2, pp. 88-93, February 2010.

[9] L. Doyle, J. McMenamy, and T. K. Forde, "Regulating for carrier aggregation \& getting spectrum management right for the longer term," in DYSPAN. IEEE, 2012, pp. 10-20.

[10] 3GPP, "Evolved Universal Terrestrial Radio Access (E-UTRA) Base Station (BS) radio transmission and reception (Release 8)," 3rd Generation Partnership Project (3GPP), TS 36.104, Jul. 2012. [Online]. Available: http://www.3gpp.org/ftp/Specs/ archive/36_series/36.104/36104-8d0.zip

[11] S. Borst, A. Mandelbaum, and M. I. Reiman, "Dimensioning large call centers," Operations Research, vol. 52, no. 1, pp. 1734, 2004.

[12] D. L. Iglehart, "Limiting diffusion approximations for the many server queue and the repairman problem," Journal of Applied Probability, vol. 2, no. 2, pp. 429-441, 1965.

[13] S. Halfin and W. Whitt, "Heavy-traffic limits for queues with many exponential servers," Operations research, vol. 29, no. 3 , pp. 567-588, 1981.

[14] S. Zeltyn and A. Mandelbaum, "Call centers with impatient customers: many-server asymptotics of the $\mathrm{M} / \mathrm{M} / \mathrm{n}+\mathrm{G}$ queue," Queueing Systems, vol. 51, no. 3-4, pp. 361-402, 2005.

[15] Z. Shen, A. Papasakellariou, J. Montojo, D. Gerstenberger, and F. Xu, "Overview of 3GPP LTE-advanced carrier aggregation for 4G wireless communications," Communications Magazine, IEEE, vol. 50, no. 2, pp. 122-130, February 2012.

[16] M. N. Alotaibi and M. A. Sirbu, "Spectrum aggregation technology: Benefit-cost analysis and its impact on spectrum value." TPRC, 2011.

[17] M. Weiss, P. Krishnamurthy, L. E. Doyle, and K. Pelechrinis, "When is electromagnetic spectrum fungible?" in DYSPAN. IEEE, 2012, pp. 349-357.

[18] H. Bolcskei, R. Nabar, O. Oyman, and A. Paulraj, "Capacity scaling laws in MIMO relay networks," Wireless Communications, IEEE Transactions on, vol. 5, no. 6, pp. 1433-1444, June 2006.

[19] P. Gupta and P. Kumar, "The capacity of wireless networks," Information Theory, IEEE Transactions on, vol. 46, no. 2, pp. $388-404$, March 2000.

[20] L.-L. Xie and P. Kumar, "A network information theory for wireless communication: scaling laws and optimal operation," Information Theory, IEEE Transactions on, vol. 50, no. 5, pp. 748-767, May 2004.

[21] A. Ozgur, O. Leveque, and D. Tse, "Hierarchical cooperation achieves optimal capacity scaling in ad hoc networks," Information Theory, IEEE Transactions on, vol. 53, no. 10, pp. 35493572, Oct 2007.
[22] E. Kavurmacioglu, M. Alanyali, and D. Starobinski, "Demandinvariant price relationships and market outcomes in competitive private commons," ACM Transactions on Internet Technology (TOIT), vol. 14, no. 2-3, p. 15, 2014.

[23] K. P. Jagannathan, I. Menache, E. Modiano, and G. Zussman, "Non-cooperative spectrum access: the dedicated vs. free spectrum choice." in MobiHoc. ACM, 2011, p. 10.

[24] G. Kasbekar and S. Sarkar, "Spectrum pricing games with arbitrary bandwidth availability probabilities," in ISIT, August 2011, pp. $2711-2715$.

[25] L. Duan, J. Huang, and B. Shou, "Competition with dynamic spectrum leasing," CoRR, vol. abs/1003.5517, 2010.

[26] D. Niyato and E. Hossain, "Competitive pricing for spectrum sharing in cognitive radio networks: Dynamic game, inefficiency of Nash equilibrium, and collusion," Selected Areas in Cоттиnications, IEEE Journal on, vol. 26, no. 1, pp. 192 -202, 2008.

[27] S. Sengupta and M. Chatterjee, "An economic framework for dynamic spectrum access and service pricing," Networking, IEEE/ACM Transactions on, vol. 17, no. 4, pp. 1200-1213, 2009.

[28] Y. Xing, R. Chandramouli, and C. Cordeiro, "Price dynamics in competitive agile spectrum access markets," Selected Areas in Communications, IEEE Journal on, vol. 25, no. 3, pp. $613-621$, 2007.

[29] T. Bonald, "The Erlang model with non-Poisson call arrivals," ACM SIGMETRICS Performance Evaluation Review, vol. 34, no. 1, pp. 276-286, 2006.

[30] — "Insensitive queueing models for communication networks," in Proceedings of the 1st International Conference on Performance Evaluation Methodolgies and Tools, ser. Valuetools '06. New York, NY, USA: ACM, 2006.

[31] G. Zeng, "Two common properties of the Erlang-B function, Erlang-C function, and Engset blocking function," Mathematical and Computer Modelling, vol. 37, no. 1213, pp. 1287 - 1296, 2003.

[32] "LTE in a nutshell," http://www.tsiwireless.com/docs/ whitepapers/LTE\%20in\%20a\%20Nutshell\%20-\%20Physical\% 20Layer.pdf, Telesystem Innovations, 2010.

[33] D. R. Smith and W. Whitt, "Resource sharing for efficiency in traffic systems," Bell System Technical Journal, vol. 60, no. 1, pp. 39-55, 1981.

[34] D. L. Jagerman, "Some properties of the Erlang loss function," Bell System Tech. J, vol. 53, no. 3, pp. 525-551, 1974.

[35] E. Kavurmacioglu and D. Starobinski, "Network dimensioning with carrier aggregation," Boston University, CISE Tech. Report 2015-IR-0001, April 2015. [Online]. Available: http: //www.bu.edu/phpbin/cise/download.php?publication_id=1266

[36] "Mobile spectrum dashboard," http://reboot.fcc.gov/reform/ systems/spectrum-dashboard, The Federal Communications Commission.

[37] T. Bonald and J. W. Roberts, "Internet and the Erlang formula," ACM SIGCOMM Computer Communication Review, vol. 42, no. 1, pp. 23-30, 2012.

[38] E. Kavurmacioglu, M. Alanyali, and D. Starobinski, "Competition in secondary spectrum markets: Price war or market sharing?" in DYSPAN. IEEE, 2012, pp. 440-451.

[39] V. Valenta, R. Marsalek, G. Baudoin, M. Villegas, M. Suarez, and F. Robert, "Survey on spectrum utilization in Europe: Measurements, analyses and observations," in CROWNCOM. IEEE, 2010, pp. 1-5. 